\title{
Anti-tumor response induced by immunologically modified carbon nanotubes and laser irradiation using rat mammary tumor model
}

\author{
Joseph T. Acquaviva III*, Cody F. Bahavar*, Feifan Zhou*, Xiaosong Li*, \\ Eric W. Howard ${ }^{\S}$, Liz C. Bullen ${ }^{\S}$, Ricardo P. Silvy ${ }^{\dagger}$ and Wei R. Chen*, \\ *Biophotonics Research Laboratory \\ Center for Interdisciplinary Biomedical Education and Research \\ University of Central Oklahoma, Edmond, Oklahoma 73034, USA \\ ${ }^{\dagger}$ SouthWest Nano Technologies, Norman, Oklahoma 73071, USA \\ \$Department of Oncology \\ The First Affiliated Hospital of Chinese \\ PLA General Hospital, Fucheng Road 51 \\ Beijing, P. R. China \\ $\S_{\text {University of Oklahoma Health Sciences Center }}$ \\ 940 Stanton. L. Young Blvd., BMSB Rm.513 Oklahoma City \\ Oklahoma 73104, USA \\ 『wchen@uco.edu
}

Received 5 January 2015

Accepted 8 April 2015

Published 14 May 2015

\begin{abstract}
The ideal treatment modality for metastatic cancer would be a local treatment that can destroy primary tumors while inducing an effective systemic anti-tumor response. To this end, we developed laser immunotherapy, combining photothermal laser application with an immunoadjuvant for the treatment of metastatic cancer. Additionally, to enhance the selective photothermal effect, we integrated light-absorbing nanomaterials into this innovative treatment. Specifically, we developed an immunologically modified carbon nanotube combining single-walled carbon nanotubes (SWNTs) with the immunoadjuvant glycated chitosan (GC). To determine the effectiveness of laser irradiation, a series of experiments were performed using two different irradiation durations -5 and 10 min. Rats were inoculated with DMBA- 4 cancer cells, a metastatic cancer cell line. The treatment group of rats receiving laser irradiation for $10 \mathrm{~min}$ had a $50 \%$ longterm survival rate without residual primary or metastatic tumors. The treatment group of rats receiving laser irradiation for $5 \mathrm{~min}$ had no long-term survivors; all rats died with multiple metastases at several distant sites. Therefore, Laser+SWNT-GC treatment with $10 \mathrm{~min}$ of laser
\end{abstract}

TCorresponding author.

This is an Open Access article published by World Scientific Publishing Company. It is distributed under the terms of the Creative Commons Attribution 3.0 (CC-BY) License. Further distribution of this work is permitted, provided the original work is properly cited. 
J. T. Acquaviva III et al.

irradiation proved to be effective at reducing tumor size and inducing long-term anti-tumor immunity.

Keywords: Anti-tumor immune response; laser immunotherapy; single-walled carbon nanotubes; glycated chitosan; laser irradiation.

\section{Introduction}

Metastases continue to be one of the primary reasons for cancer patient deaths and treatment failures. The systemic and recurrent nature of cancers should be the primary focus when developing new treatment modalities against cancers. Recent studies suggest that the immune system can be activated against cancer cells. ${ }^{1-3}$ In particular, the secretion of cytokines, reduction of the tumor's defense systems, activation of dendritic cells, and proliferation of $\mathrm{T}$ and $\mathrm{B}$ cells, are of vital importance for inducing a systemic anti-tumor immunological response. However, the method of activating the immune system is one of the many challenges facing current immunotherapies.

Laser immunotherapy was developed for the systemic treatment of metastatic cancers by synergistically combining thermal laser irradiation with immunological stimulation. ${ }^{4}$ Studies have shown that thermal laser irradiation on cancer cells can immediately increase cytokine production and tumor antigen release, which could aid in activation of the immune system. ${ }^{5}$ The immunoadjuvant glycated chitosan (GC) was formulated to enhance the activation of the immune system. ${ }^{6} \mathrm{GC}$ is biocompatible, water soluble, and capable of increasing the concentration of cytokines as well as activating immune cells. ${ }^{7,8}$ Additionally, GC can be used as a surfactant. With the synergistic combination of thermal laser irradiation and immunological stimulation, laser immunotherapy is capable of inducing an effective anti-tumor immunological response in late stage metastatic cancer patients. ${ }^{9}$

Recently, nanostructures have become increasingly popular in nearly all aspects of scientific research, particularly in drug delivery. One such nanostructure is the single-walled carbon nanotubes (SWNTs). SWNTs are composed of carbon atoms double bonded together creating a graphene single layered tube. ${ }^{10}$ The three-dimensional shape of the SWNTs allow the nanostructure to have an increased absorption of light at specific wavelengths. ${ }^{11}$ Specifically, SWNTs are chiral and can be synthesized in an $(\mathrm{m}, \mathrm{n})$ fashion, which influences their enhanced light absorption property. Furthermore, due to the polarity of the SWNTs and the increased mitochondrial membrane potential in cancer cells, the nanostructure can cross cell membranes and enter into the mitochondria of cancer cells. ${ }^{12}$ Therefore, SWNTs can not only be used as a drug delivery vehicle, but also as a locally selective light absorption enhancing agent.

The goal of this work is to determine the efficacy of this novel nanotechnology-based laser immunotherapy on treating metastatic tumors, and to determine the effectiveness of laser irradiation on treating tumors injected with SWNT-GC. Furthermore, we conjugated the SWNTs to a nonbiological active surfactant, sodium cholate $(\mathrm{NaC})$, to determine how thermal laser application alone induces anti-tumor reaction. Rats with an aggressive tumor model were intratumorally injected with SWNT-GC, and, $2 \mathrm{~h}$ later, received topical laser treatment on the tumor for 5 or $10 \mathrm{~min}$.

\section{Materials and Methods}

\subsection{Single-walled carbon nanotube}

We used SWNTs produced by the CoMoCAT method obtained from Southwestern Nanotechnologies, Inc. (Norman, Oklahoma). The production of SWNTs using the CoMoCAT method was performed using a silica support and a bimetallic catalyst prepared from cobalt nitrate and ammonium heptamolybdate precursors. Total metallic loading in the catalyst was between 2 and 3 wt.\%, with a Co:Mo molar ratio of 1:3. Prior to exposure to the $\mathrm{CO}$ feedstock, the catalyst was heated to $500^{\circ} \mathrm{C}$ and then to $750^{\circ} \mathrm{C}$ under a constant flow of helium gas. Utilizing a fluidized bed reactor under a flow of pure $\mathrm{CO}$ at 5 atmospheres total pressure, a $\mathrm{CO}$ disproportionation reaction was used to produce SWNTs. The material grown by this method remained mixed with the spent catalyst, containing silica support and the Co and Mo species. To remove the silica support from 
this mixture, the solid product was suspended in a stirred $10-20 \mathrm{wt} . \% \mathrm{HF}$ solution for $10 \mathrm{~min}$ to $3 \mathrm{~h}$ at $25^{\circ} \mathrm{C}$. This suspension was then filtered through a polytetrafluoroethylene (PTFE) membrane with a pore size of $0.2 \mu \mathrm{m}$ and washed with deionized water to a neutral $\mathrm{pH} .^{13-15}$

\subsection{Immunologically modified carbon nanotube}

GC was prepared and obtained as previously described. ${ }^{4} 4 \mathrm{mg}$ SWNTs were placed in a $50 \mathrm{~mL}$ test tube. Then, $8 \mathrm{~mL}$ of $0.5 \% \mathrm{GC}$ was dispensed in the test tube. The mixture was placed in an ice bath and sonicated for $2 \mathrm{~h}$ at $30 \mathrm{~min}$ intervals, and then centrifuged at $10,000 \mathrm{rpm}$ for $10 \mathrm{~min}$. The supernatant was then collected and stored at $4{ }^{\circ} \mathrm{C}$.

\subsection{Determination of chirality, diameter combination, and optical absorbance of $S W N T s$}

Using a solution of SWNT-GC (3 mg SWNTs in $7 \mathrm{~mL} 0.5 \%$ GC), a one-dimensional emission spectra probed at $532 \mathrm{~nm}, 636 \mathrm{~nm}, 671 \mathrm{~nm}$, and $783 \mathrm{~nm}$ wavelengths for excitation were collected using an NS2 Nanospectralyzer (Applied Nanofluorescence, LLC). These emission spectra were used to fit a model to a two-dimensional photoluminescence map in order to estimate the semiconducting chirality composition and diameter distribution of the SWNTs solution. Using a different SWNT-GC solution (15 mg SWNTs in $7 \mathrm{~mL} 0.5 \% \mathrm{GC}$ ) and its dilutions (10- or 100-fold), Raman spectra excited at $532 \mathrm{~nm}$ and UV-Vis optical absorbance spectra were also acquired using the NS2 equipment.

\section{4. $S W N T-N a C$}

About $2 \mathrm{mg}$ of sodium cholate was completely dissolved in $98 \mathrm{~mL}$ of deionized $\mathrm{H}_{2} \mathrm{O}$ to produce a $2 \% \mathrm{NaC}$ solution. Then, $10 \mathrm{~mL}$ of the $2 \% \mathrm{NaC}$ solution was dispensed into a $50 \mathrm{~mL}$ centrifuge tube containing $5 \mathrm{mg}$ of SWNTs. The SWNT-NaC mixture was placed in an ice bath and sonicated for $2 \mathrm{~h}$ at $30 \mathrm{~min}$ intervals.

\subsection{Animal treatment}

A 0.1 -mL solution of DMBA-4 cells $\left(1 \times 10^{5}\right)$ were injected into the flank region of 6-8 weeks old female Wistar Furth rats. The rats were treated 1014 days after tumor inoculation, when tumor volume was approximately $0.5-1 \mathrm{~cm}^{3}$. Tumor-bearing rats were separated into five different treatment groups: control $n=12$, laser for 10 min only $n=8$, SWNT$\mathrm{NaC}$ with 10 min of laser irradiation $n=12$, as well as Laser+SWNT-GC with 5 min or 10 min of laser irradiation, $n=8$ and $n=10$, respectively. $2 \mathrm{~h}$ prior to laser irradiation, the hair on the tumor was shaved and a $200 \mu \mathrm{L}$ solution of SWNT-GC (100$1000 \mu \mathrm{g})$ was injected directly into the center of the primary tumor. Laser irradiation encompassed the primary tumor and $0.5-1 \mathrm{~cm}$ of the surrounding skin, and was delivered through an optical fiber noninvasively at $980 \mathrm{~nm}$ with a power density of $1.0 \mathrm{~W} / \mathrm{cm}^{2}$ for either $5 \mathrm{~min}$ or $10 \mathrm{~min}$. The rats were anesthetized with isoflurane during treatment. The rats were observed and the primary tumor and metastatic tumors were measured twice a week after treatment. Surviving rats were re-challenged 100 days after treatment with an 0.1-mL injection of $1 \times$ $10^{6}$ DMBA-4 cells.

\section{Results}

\subsection{Size distribution of $S W N T s$ and optical absorbance}

As shown in Fig. 1(a), the SWNTs had a $(\mathrm{n}, \mathrm{m})$ ratio distribution of: $23 \%(6,5), 21 \%$ $(7,3), 9 \%(10,3), 9 \%(7,6), 8 \%(8,4)$, and $32 \%$ (other). The average diameter from fluorescence analysis of the SWNTs was $0.856 \mathrm{~nm}$ [Fig. 1(b)]. Due to the $(\mathrm{n}, \mathrm{m})$ configuration, SWNTs had an increased absorbance peak near $980 \mathrm{~nm}$, which increased proportionally with the amount of SWNTs (Fig. 2).

\subsection{Anti-tumor effects of treatment}

SWNT-GC with laser irradiation for $5 \mathrm{~min}$ was capable of inducing complete primary tumor regression in one out of eight rats (12.5\%) (Fig. 3). However, SWNT-GC with 10 min of laser irradiation was capable of inducing complete primary tumor regression in 7 out of 10 rats (70\%) (Fig. 3). In addition, SWNT-GC with $10 \mathrm{~min}$ of laser irradiation was capable of inducing complete metastatic tumor regression at the left and right axillae (Fig. 4). Furthermore, rats treated by SWNT-GC with 10 min of laser irradiation had a $50 \%$ (5 out of 


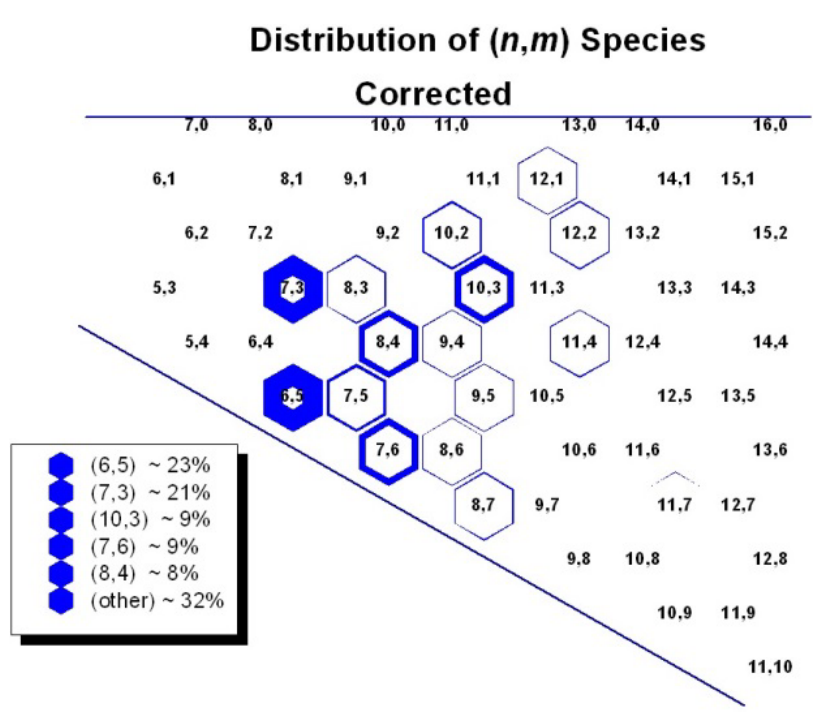

(a)

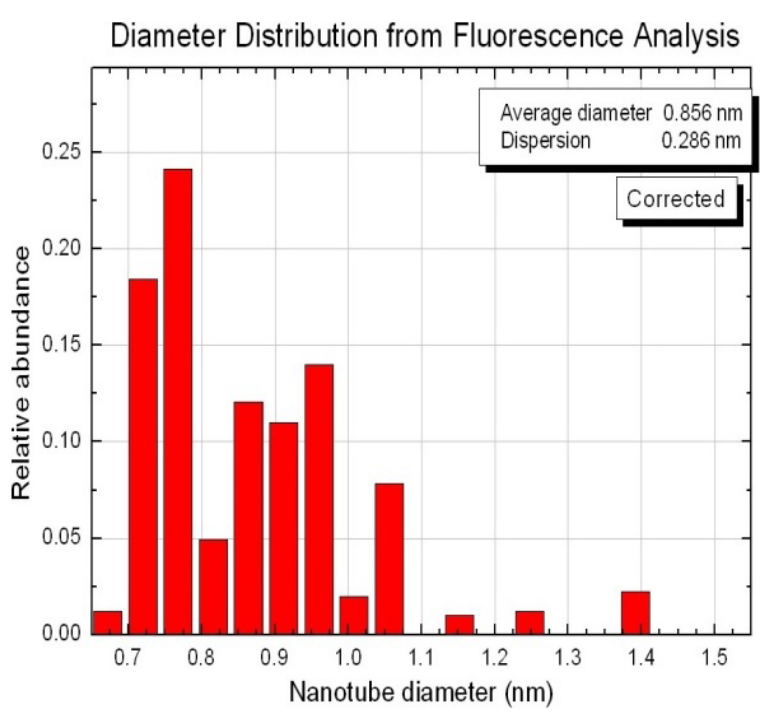

(b)

Fig. 1. Determination of chirality and diameter of SWNTS. Using a SWNT-GC solution (3 mg SWNTs and $7 \mathrm{~mL} 0.5 \%$ GC), onedimensional emission spectra excited at 532,636,671, and $783 \mathrm{~nm}$ wavelengths were collected. The emission spectra were used to fit a model to a two-dimensional photoluminescence map (a) in order to estimate the semiconducting chirality composition and diameter distribution of the SWNT solution (b).

10) long-term survival rate, while Laser+SWNTGC with 5 min of laser irradiation produced no longterm survivors (Fig. 5). Additionally, 10 min of laser irradiation alone was capable of inducing a $12.5 \%$ survival rate and SWNT-NaC with 10 min of laser irradiation was capable of inducing $22 \%$ survival rate. A total of 100 days after treatment of all the surviving rats were re-challenged with 10 times the orignal amount of DMBA-4 tumor cells. All the surviving rats failed the re-challenge except the SWNT-GC with $10 \mathrm{~min}$ of laser irradation, which had a $100 \%$ survival rate (figure not shown).

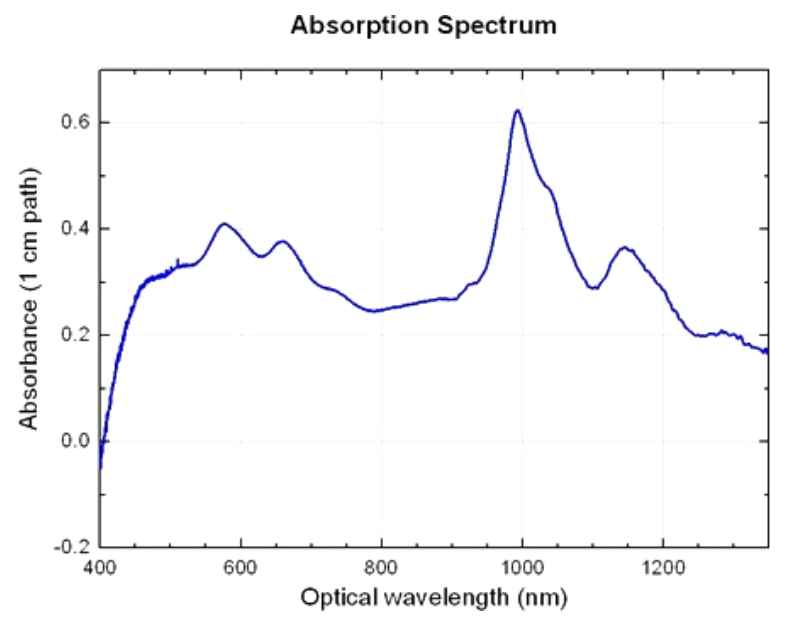

(a)

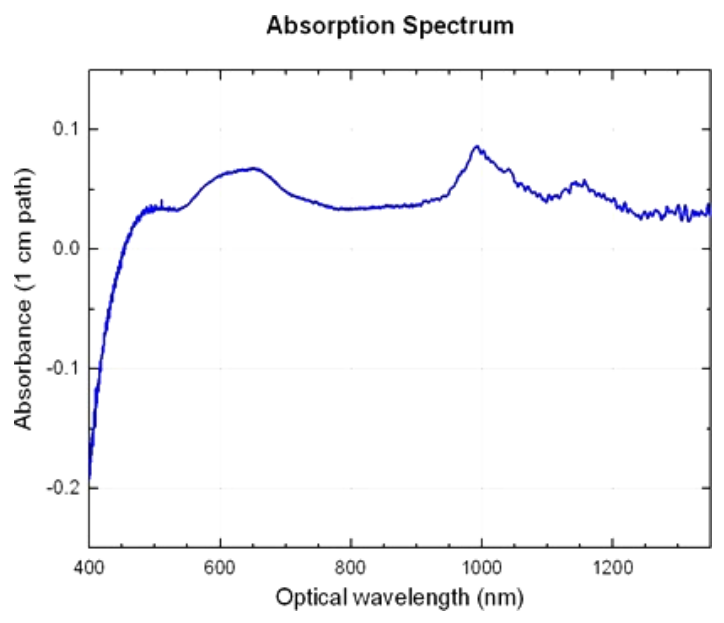

(b)

Fig. 2. Optical absorbency of SWNTs. Raman spectra of different SWNT-GC solutions were collected from NS2 equipment at $532 \mathrm{~nm}$ and UV-Vis optical absorbance. (a) Raman spectrum of solution with lower SWNT concentration (3 mg SWNT in $7 \mathrm{~mL}$ GC, diluted 10 folds); (b) Raman spectrum of solution with lower SWNT concentration (3 mg SWNT in $7 \mathrm{~mL}$ GC, diluted 100 folds). (c) Raman spectrum of solution with higher SWNT concentration (15 mg SWNT in $7 \mathrm{~mL}$ GC, diluted 10 folds); (d) Raman spectrum of solution with higher SWNT concentration (15 mg SWNT in $7 \mathrm{~mL}$ GC, diluted 100 folds). 


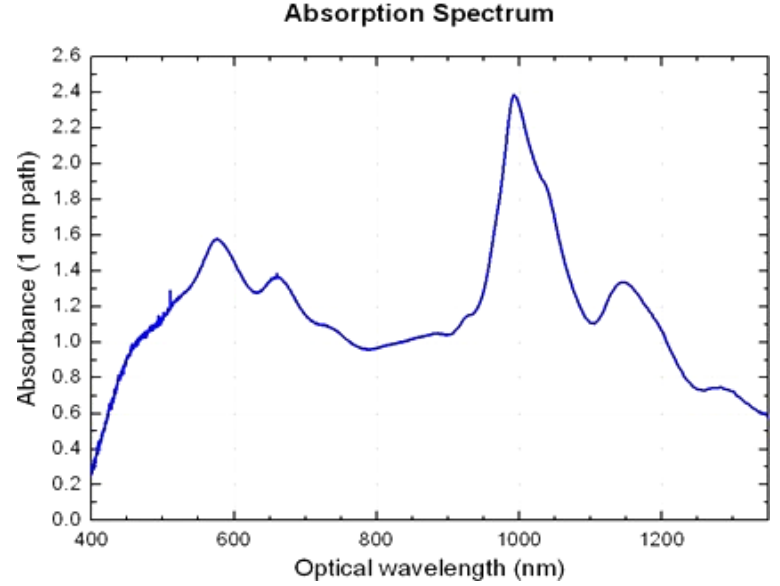

(c)

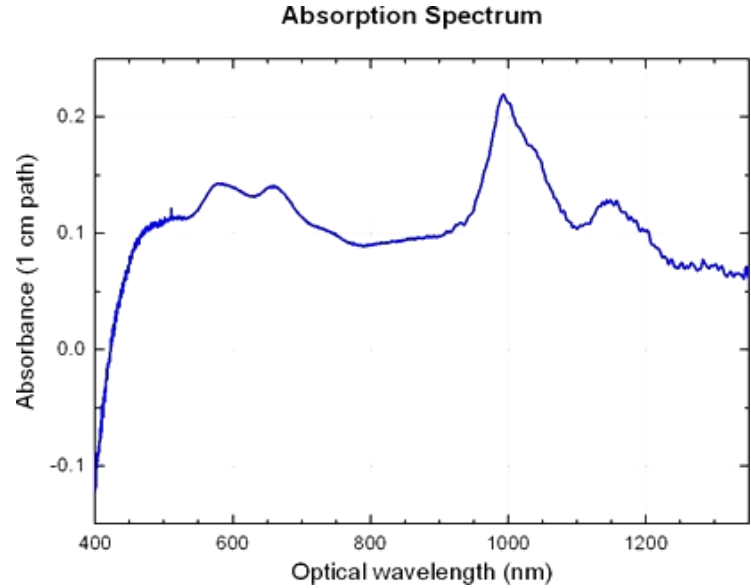

(d)

Fig. 2. (Continued)

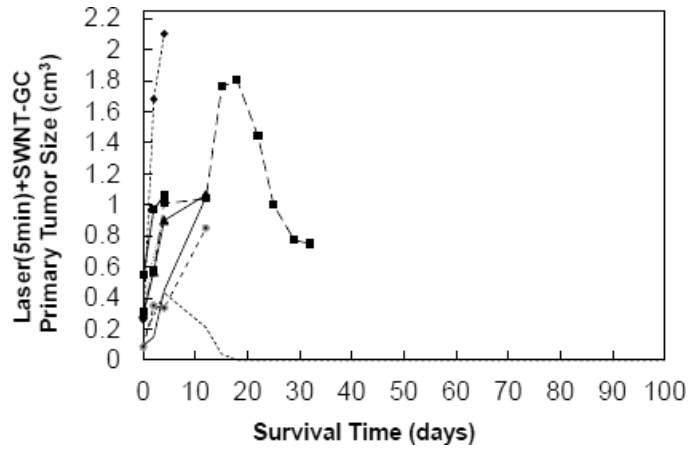

(a)

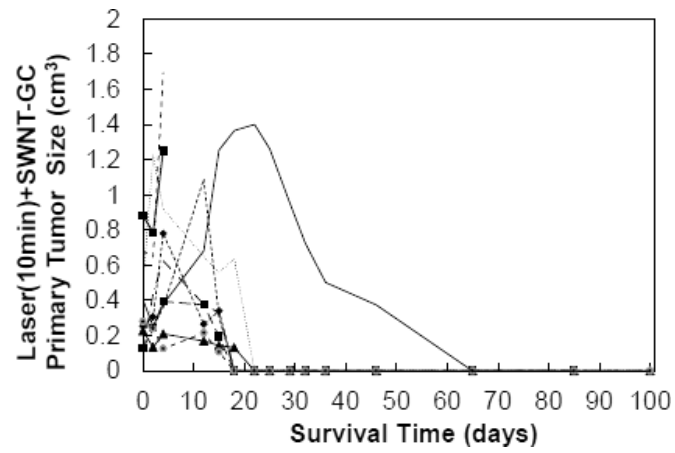

(b)

Fig. 3. Regression of primary tumors. Tumor-bearing rats were treated 10-14 days after tumor inoculation, when tumor volume was approximately $0.5 \mathrm{~cm}^{3}$. A $200-\mu \mathrm{l}$ solution of SWNT-GC $(100-1000 \mu \mathrm{g})$ was injected directly into the center of the primary tumor $2 \mathrm{~h}$ prior to laser irradiation, with a power density of $1.0 \mathrm{~W} / \mathrm{cm}^{2}$ for either 5 or $10 \mathrm{~min}$. (a) Volumetric measurements of primary tumors receiving SWNT-GC and 5 min of laser irradiation. (b) Volumetric measurements of primary tumors receiving SWNT-GC and $10 \mathrm{~min}$ of laser irradiation.

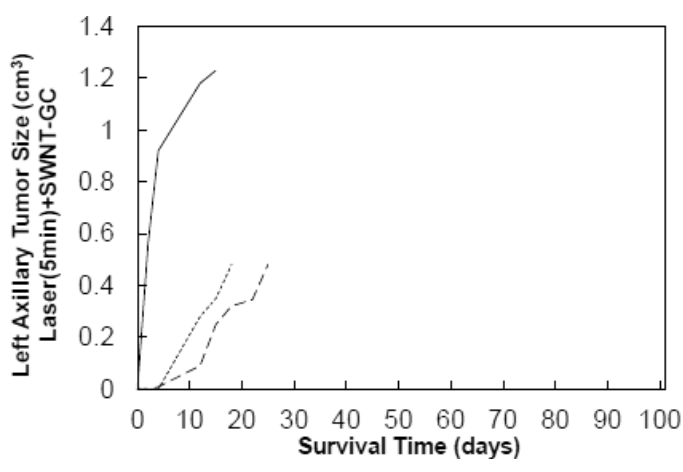

(a)

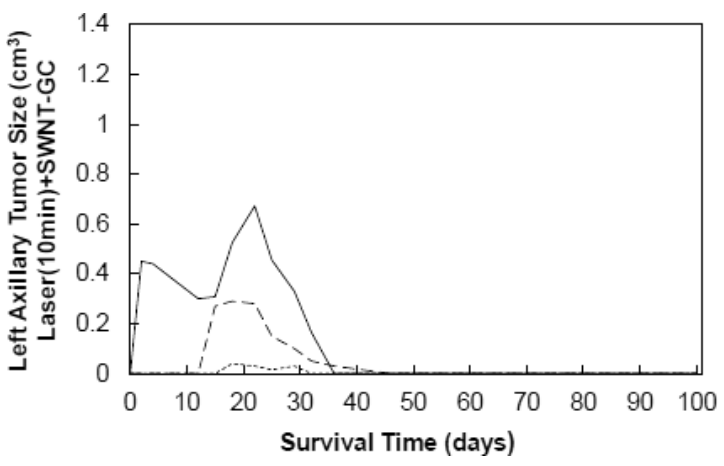

(b)

Fig. 4. Regression of metastatic tumors. Primary tumors in rats were treated 10-14 days after tumor inoculation, when tumor volume was approximately $0.5 \mathrm{~cm}^{3}$. Untreated metastatic tumors were measured twice per week. (a) Left axillary metastases of rats receiving SWNT-GC with $5 \mathrm{~min}$ of laser irradiation. (b) Left axillary metastases of rats receiving SWNT-GC with 10 min of laser irradiation. (c) Right axillary metastases of rats receiving SWNT-GC with 5 min of laser irradiation. (d) Right axillary metastases of rats receiving SWNT-GC with 10 min of laser irradiation. 


\section{J. T. Acquaviva III et al.}

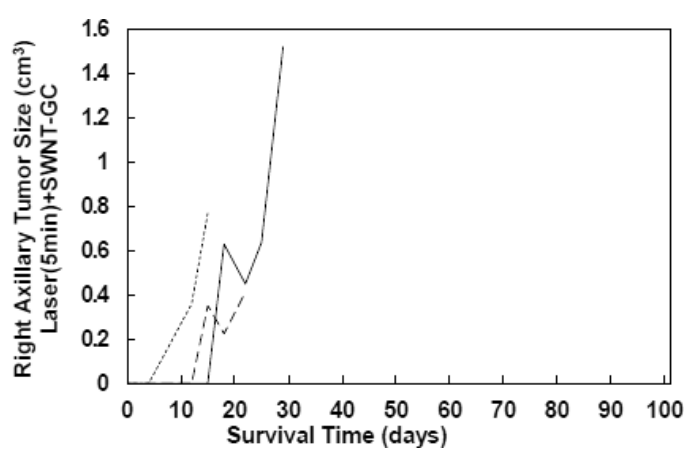

(c)

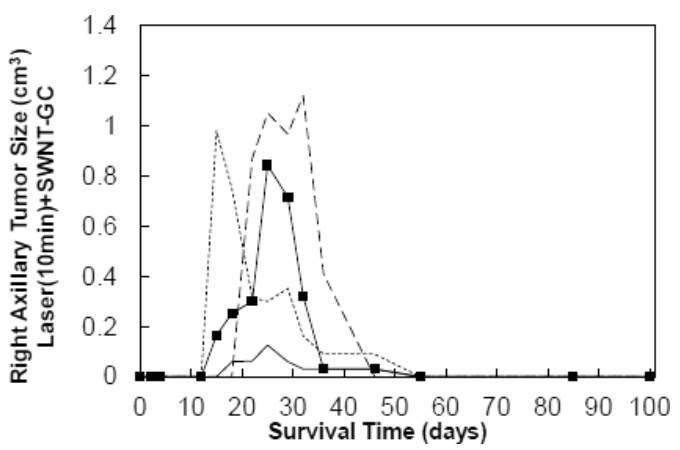

(d)

Fig. 4. (Continued)

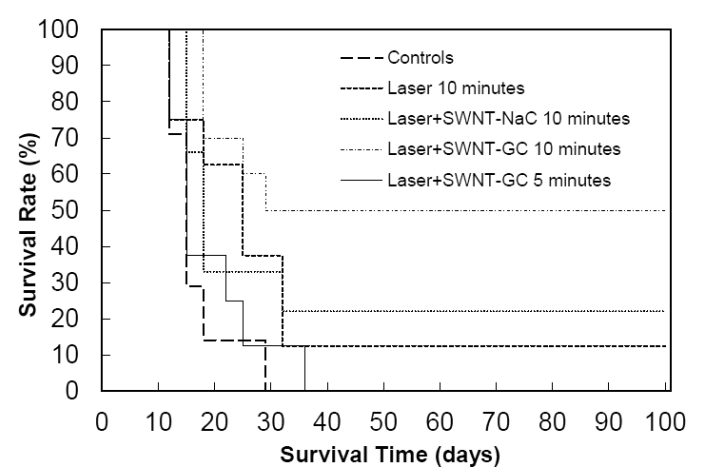

Fig. 5. Overall survival rates. Primary tumors in rats were treated 10-14 days after tumor inoculation, when tumor volume was approximately $0.5 \mathrm{~cm}^{3}$. The treated rats were observed up to 100 days. Rats receiving Laser+SWNT-GC for $10 \mathrm{~min}$ had a $50 \%$ long-term survival rate, rats receiving Laser+ SWNT-GC for $5 \mathrm{~min}$ had a $0 \%$ survival rate, rats receiving Laser+SWNT-NaC for $10 \mathrm{~min}$ had a $22 \%$ long-term survival rate, and rats receiving laser only treatment for $10 \mathrm{~min}$ had a $12.5 \%$ long-term survival rate.

\section{Discussion}

Immunotherapies are beginning to show promise in the treatment of cancers..$^{3,4,7-9}$ When using laser irradiation as part of a cancer treatment, problems can arise when the tumor is deeply embedded in tissue or under heavily pigmented skin, as the laser light is not effectively absorbed and distributed evenly in the tumor. ${ }^{16,17}$ Treatment modalities utilizing laser irradiation require other methods to overcome such obstacles. There are many potential options, including absorption-enhancing dyes, invasive laser fibers, and nanomaterials. We believe that incorporating nanomaterials into laser immunotherapy could enhance the delivery and distribution of laser light. Other approaches, such as interstitial laser irradiation, could further enhance laser immunotherapy.

Previously, laser immunotherapy utilized a laserabsorbing dye known as indocyanine green (ICG). A similar experiment utilizing Laser+ICG-GC and DMBA-4 tumor-bearing rats resulted in a $25 \%$ survival rate. ${ }^{18}$ The current study increased animal survival by taking advantage of the geometrical configuration of the SWNTs to allow better delivery and distrubution of the $980 \mathrm{~nm}$ laser light. Additionally, this study investigated the anti-tumor effects of SWNTs induced when not conjugated to an immunoadjuvant and laser irradiation was adminstered. This study concluded that while the SWNTs enhanced the anti-tumor effect, they were not capable of inducing tumor immunity when the surviving rats from the SWNT-NaC with 10 min of laser irradiation were re-challenged with the DMBA-4 tumor cells.

One of the problems facing immunotherapies is the effective activation of the innate and adaptive arms of the immune system. One possible mechanism could be utilization of heat shock proteins (HSP). Studies show that HSP70, harvested from tumor cells, can induce tumor immunity in mice inoculated with identical tumor cells. ${ }^{19}$ Furthermore, an HSP gp96-engineered vaccine induced $\mathrm{CD}^{+} \mathrm{T}$-cell anti-tumor response, as well as proliferation of natural killer cells in a large percentage of patients in a previous clinical trial. ${ }^{20}$ Interestingly, various studies suggest that the mechanism of cell death is paramount to the release of HSP - necrotic cell death releases HSP while cell death via apoptosis may not. ${ }^{21,22}$ We believe that laser irradiation induces HSP release from tumor cells. By optimizing the laser irradiation parameters, we can 
induce necrotic cell death, leading to a more robust immunological anti-tumor response. We believe the required time to induce these $\mathrm{HSP}$ at the power densities used in these experiments was more than 5 min. Furthermore, we believe this is the reason why anti-tumor effects were seen in all groups treated with a combination of laser irradiation for $10 \mathrm{~min}$.

The effects of different durations of laser irradiation on inducing anti-tumor activities in the SWNTGC laser immunotherapy system are very important. In this work, we demonstrated that $10 \mathrm{~min}$ of laser irradiation, as opposed to $5 \mathrm{~min}$, at $980 \mathrm{~nm}$ with a power density of $1.0 \mathrm{~W} / \mathrm{cm}^{2}$, with administration of SWNT-GC as the light-absorbing immunoadjuvant, was capable of inducing regression of primary and metastatic tumors while also inducing long-term tumor immunity. We believe the longer duration of laser irradiation in this study yielded a higher temperature in the tumor, possibly inducing necrotic cell death, allowing for a more effective anti-tumor response. Furthermore, while the results suggest that Laser and Laser $+\mathrm{NaC}$ were capable of inducing tumor regression, the anti-tumor response was not strong enough to induce tumor immunity without the aid of an immunoadjuvant.

\section{Acknowledgments}

This study was supported by grants from the US National Institutes of Health (R21 EB0155091-01), Beijing Natural Science Foundation (4153064), and from Beijing Nova program (Z131107000413104).

\section{References}

1. D. M. Pardoll, S. L. Topalian, "The role of CD4 T cell responses in antitumor immunity," Curr. Opin. Immunol. 10(5), 588-594 (1998).

2. A. Porgador, D. Snyder, E. Gilboa, "Induction of antitumor immunity using bone marrow-generated dendritic cells," J. Immunol. 156(8), 2918-2926 (1996).

3. W. R. Chen, W. Zhu, J. R. Dynlacht, H. Liu, R. E. Nordquist, "Long-term tumor resistance induced by laser photo-immunotherapy," Int. J. Cancer 81(5), 808-812 (1999).

4. W. R. Chen, R. Carubelli, H. Liu, R. E. Nordquist, "Laser immunotherapy," Mol. Biotechnol. 25(1), $37-43(2003)$.

5. J. O. Funk, A. Kruse, H. Kirchner, "Cytokine production after helium neon laser irradiation in cultures of human peripheral blood mononuclear cells," Photochem. Photobiol. B Biol. 16(3), 347-355 (1992).

6. S. Song, F. Zhou, R. E. Nordquist, R. Carubelli, H. Lui, W. R. Chen, "Glycated chitosan as a new non-toxic immunological stimulant," Immunopharmacol. Immunotoxicol. 31(2), 202-208 (2009).

7. F. Zhou, X. Li, S. Song, J. T. Acquaviva III, R. F. Wolf, E. W. Howard, W. R. Chen, "Anti-tumor responses induced by laser irradiation and immunological stimulation using a mouse mammary tumor model," J. Innov. Opt. Health Sci. 6(04), 1350039 (2013).

8. W. R. Chen, H. Liu, J. A. Nordquist and R. E. Nordquist, "Tumour cell damage and leucocyte infiltration after laser immunotherapy treatment," Lasers Med. Sci. 15(1), 43-48 (2000).

9. L. Xiaosong, G. L. Ferrel, M. C. Guerra, T. Hode, J. A. Lunn, O. Adalsteinsson, R. E. Nordquist, H. Liu, W. R. Chen, "Preliminary safety and efficacy results of laser immunotherapy for the treatment of metastatic breast cancer patients," Photochem. Photobiol. Sci. 10(5), 817-821 (2011).

10. A. Bianco, K. Kostarelos and M. Prato, "Applications of carbon nanotubes in drug delivery," Curr. Opin. Chem. Biol. 9(6), 674-679 (2005).

11. S. M. Bachilo, M. S. Strano, C. Kittrell, R. H. Hauge, R. E. Smalley, B. R. Weisman, "Structureassigned optical spectra of single-walled carbon nanotubes," Science 298(5602), 2361-2366 (2002).

12. F. Zhou, D. Xing, B. Wu, S. Wu, Z. Ou, W. R. Chen, "New insights of transmembranal mechanism and subcellular localization of noncovalently modified single-walled carbon nanotubes," Nano Lett. 10(5), 1677-1681 (2010).

13. B. Kitiyanan, W. E. Alvarez, J. H. Harwell, D. E. Resasco, "Controlled production of single-wall carbon nanotubes by catalytic decomposition of $\mathrm{CO}$ on bimetallic Co-Mo catalysts," Chem. Phys. Lett. 317, 497-503 (2000).

14. S. M. Bachilo, L. Balzano, J. E. Herrera, F. Pompeo, D. E. Resasco, R. B. Weisman, "Narrow $(n, m)$-distribution of single-walled carbon nanotubes grown using a solid supported catalyst," J. Am. Chem. Soc. 125, 11186-11187 (2003).

15. G. Lolli, L. Zhang, L. Balzano, N. Sakulchaicharoen, Y. Tan, D. E. Resasco, "Tailoring $(n, m)$ structure of single-walled carbon nanotubes by modifying reaction conditions and the nature of the support of CoMo," Catal. J. Phys. Chem. B 110, 2108-2115 (2006).

16. C. A. Bailey, T. M. Cowan, V. G. Liu, E. C. Lemley, W. R. Chen, "Optimization of selective hyperthermia," Biomed. Opt. 9(3), 648-654 (2004).

17. M. B. Vrouenraets, G. W. M. Visser, G. B. Snow, V. Dongen, "Basic principles, applications in oncology 
and improved selectivity of photodynamic therapy," Anticancer Res. 23, 505-522 (2003).

18. W. R. Chen, S. W. Jeong, M. D. Lucroy, R. F. Wolf, E. W. Howard, H. Liu, R. E. Nordquist, "Induced antitumor immunity against DMBA-4 metastatic mammary tumors in rats using laser immunotherapy," Int. J. Cancer 107(6), 1053-1057 (2003).

19. H. Udono, P. K. Srivastava, "Heat shock protein 70associated peptides elicit specific cancer immunity," J. Exp. Med. 178(4), 1391-1396 (1993).

20. S. Janetzki, D. Palla, V. Rosenhauer, H. Lochs, J. J. Lewis, P. K. Srivastava, "Immunization of cancer patients with autologous cancer-derived heat shock protein gp96 preparations: A pilot study," Int. J. Cancer 88(2), 232-238 (2000).

21. S. Basu, R. J. Binder, R. Suto, K. M. Anderson, P. K. Srivastava, "Necrotic but not apoptotic cell death releases heat shock proteins, which deliver a partial maturation signal to dendritic cells and activate the NF-kB pathway," J. Immunol. 12(11), 1539-1546 (2000).

22. A. Melcher, S. Todryk, N. Hardwick, M. Ford, M. Jacobson, R. G. Vile, "Tumor immunogenicity is determined by the mechanism of cell death via induction of heat shock protein expression," Nat. Med. 4(5), 581-587 (1998). 